\title{
CRESCIMENTO E BIOMASSA DE INDIVÍDUOS JOVENS DE Cabralea canjerana (Vell.) Mart.
}

\author{
GROWTH AND BIOMASS OF YOUNG INDIVIDUALS OF Cabralea canjerana (Vell.) Mart.
}

\author{
Karina Lanzarin ${ }^{1}$, Frederico Dimas Fleig' ${ }^{2}$ Evandro Alcir Meyer ${ }^{3}$, Lílian Daniel Pereira ${ }^{4}$, Thomas \\ Schröder ${ }^{5}$, Gerson dos Santos Lisboa ${ }^{6}$ \\ 1, 2, 3, 4, 5 Universidade Federal de Santa Maria, Santa Maria, Rio Grande do Sul, Brasil - \\ karinalanzarin@gmail.com,dimasfleig@uol.com.br,eam.meyer@gmail.com, \\ liliandapereira@yahoo.com.br \& thomaschroder@gmail.com \\ ${ }^{6}$ Universidade Federal do Sul da Bahia, Itabuna, Bahia, Brasil-gerson.lisboa@gmail.com
}

\section{RESUMO}

Este trabalho possui como objetivo ajustar e selecionar modelos matemáticos capazes de estimar o incremento periódico em área basal, o volume e biomassa de indivíduos jovens de Cabralea canjerana (Vell.) Mart. O estudo foi desenvolvido no município de Agudo - RS, em uma área de Floresta Estacional Decidual. Para análise de crescimento foram utilizadas 19 árvores. A quantificação da biomassa foi obtida pelo método direto com 14 árvores da espécie. No cálculo de volume foram selecionadas e cubadas 12 árvores pelo método de Smalian. Na modelagem da estimativa das variáveis incremento periódico em área basal, volume, biomassa total, biomassa da madeira e biomassa foliar foram testados seis modelos matemáticos. Para avaliação das equações ajustadas foi utilizada a menor soma dos escores dos critérios estatísticos: coeficiente de determinação, erro padrão da estimativa e coeficiente de variação em percentagem, em conjunto com a análise gráfica da dispersão dos resíduos. O modelo matemático de Schumacher-Hall obteve melhor ajuste nas estimativas de incremento periódico em área basal, volume, biomassas total e da madeira. A equação definida para o incremento periódico em área basal foi $\operatorname{lnIPg}=-0,149+1,777$. InDAP-0,815. InHt. A equação de volume selecionada foi $\operatorname{lnV}=-$ 9.382+1.583. InDAP+1.064. In Ht. As equações definidas para biomassa aérea total e biomassa da madeira foram $\operatorname{lnBt}=-3,769+1,779$. InDAP+1,131 $\ln \mathrm{Ht}$ e $\operatorname{lnBm}=-3,986+1,784$. InDAP+1,198InHt, respectivamente. As equações para estimativa de biomassa foliar obtiveram estatísticas insatisfatórias e apresentaram elevados valores de coeficiente de variação em percentagem.

\section{PALAVRAS-CHAVE: Canjerana, Estimativa de biomassa, Incremento em área basal, Volume.}

\section{ABSTRACT}

This work aims to adjust and select mathematical models capable of estimating the periodic increment in basal area, the volume and biomass of young individuals of Cabralea canjerana (Vell.) Mart. The study was developed in the municipality of Agudo - RS, in a Deciduous Seasonal Forest area. For growth analysis, 19 trees were used. The biomass quantification was obtained by the direct method with 14 trees of the species. In the volume calculation 12 trees were selected and measured by the Smalian method. In the modeling of the estimation of variables: periodic increment in basal area, volume, total biomass, wood biomass and leaf biomass were tested six mathematical models. To evaluate the adjusted equations, the lowest sum of the scores of the statistical criteria was used: coefficient of determination, standard error of the estimate and coefficient of variation in percentage, together with the graphic analysis of the dispersion of the residues. The mathematical model of Schumacher-Hall obtained better adjustment in the estimates of periodic increment in basal area, volume, total biomass and wood biomass. The equation defined for the periodic increment in basal area was $\ln I P g=-0.149+$ 1.777. InDAP-0.815. InHt. The volume equation selected was $\operatorname{lnV}=-9.382+1.583 . \operatorname{InDAP}+1.064 . \operatorname{InHt}$. The equations defined for total aerial biomass and biomass of wood were $\ln B t=-3.769+1.779 . \ln D A P+$ $1.131 \mathrm{InHt}$ and $\mathrm{InBm}=-3.986+1.784 . \operatorname{lnDAP}+1.198 \mathrm{InHt}$, respectively. The equations for estimation of leaf biomass obtained unsatisfactory statistics and presented high values of coefficient of variation in percentage.

KEYWORDS: Canjerana, Estimation of biomass, Increment in basal area, Volume. 


\section{INTRODUÇÃO}

O manejo florestal sustentável é definido como administração da floresta para a obtenção de benefícios econômicos e sociais, respeitando-se os mecanismos de sustentação do ecossistema. Deixando claro que para ser sustentável, o manejo deve ser economicamente viável, ecologicamente correto e socialmente justo (SILVA, 1996).

No manejo sustentado, a floresta deve ser manejada de tal maneira que ofereça em longo prazo os mesmos benefícios que no presente (SCHNEIDER, 2008). Visandose a sustentabilidade da produção é de intrínseca importância conhecer as variáveis das florestas, como o crescimento em diâmetro, volumétrico e a produção de biomassa. Vaccaro et al. (2003) ainda comentam a importância do conhecimento do incremento das árvores a serem manejadas, na elaboração de planos de manejo em regime sustentado e salientam que no Rio Grande do Sul, existe uma carência de informações quanto ao crescimento de árvores nativas, o que impede a elaboração desses planos.

A principal característica de uma floresta nativa é a heterogeneidade. Há grande diversidade de espécies, de diferentes idades e tamanhos, não sendo viável tratar todos os indivíduos como um povoamento homogêneo. Nestes casos, o manejo deve ser realizado considerando a árvore de forma individual (CHASSOT, 2009).

As pesquisas de biomassa florestal são feitas com objetivos diversos, dentre os quais, destacam-se a quantificação da ciclagem de nutrientes, a quantificação para fins energéticos e como base de informação para estudos de sequestro de carbono. Estes estudos são de grande importância para a tomada de decisões no manejo dos recursos florestais (PÁsCOA et al., 2004).

A mensuração da biomassa pode ser efetuada utilizando métodos diretos e indiretos, sendo que o método direto consiste em cortar as árvores, separar e pesar seus elementos, enquanto que no método indireto são utilizadas funções alométricas ou imagens de satélite para realização das estimativas (WATZLAWICK, 2003).

Tipos diferentes de florestas e locais diferentes dentro da mesma tipologia florestal apresentam variações significativas na quantidade de biomassa e armazenam quantidades de carbono diferenciadas (HOUGHTON, 1994). Silveira et. al. (2008) alegam que a maioria das estimativas de biomassa consideram apenas as partes vivas das plantas acima do solo com diâmetros mínimos, e sugerem que sejam consideradas também as árvores de menor porte.
A espécie Cabralea canjerana (Vell.) Mart., popularmente denominada canjerana, é uma das árvores mais comuns e conhecidas no Rio Grande do Sul, possui extraordinária durabilidade e é considerada como uma das madeiras mais valiosas no Sul do Brasil (REITZ, 1983).

A canjerana é uma árvore que atinge de 20 a $30 \mathrm{~m}$ de altura e 70 a $120 \mathrm{~cm}$ de diâmetro à altura do peito (LORENZI, 1998). Reitz et al. (1988) menciona que a espécie possui tronco cilíndrico, levemente tortuoso e fuste curto variando de cinco a oito metros de comprimento. Carvalho (1994) menciona que a canjerana apresenta tronco cilíndrico, reto ou geralmente pouco tortuoso e fuste com até $12 \mathrm{~m}$ de comprimento.

A canjerana é uma espécie secundária tardia que pode ultrapassar 300 anos de idade, de acordo com Carvalho (1994). Reitz et al. (1988) mencionam a agressividade bastante pronunciada da canjerana sobre os capoeirões e florestas secundárias no Sul do Brasil, demonstrando, grande potencial de regeneração e dinamismo nas associações secundárias, fato muito importante na silvicultura com espécies nativas. A madeira da canjerana é moderadamente pesada $(0,65-$ $0,75 \mathrm{~g} \cdot \mathrm{cm}^{-3}$ ) e caracteriza-se por ser resistente à umidade e aos agentes degradantes, conforme descrevem Santos (1987), Reitz et al. (1988), Carvalho (1994) e Lorenzi (1998).

A espécie é utilizada para diversos fins, tais como: construção civil, obras externas e internas, dormentes, marcenaria, tábuas, moirões, carpintaria, etc. A madeira apresenta grande durabilidade quando exposta ao tempo, motivo pelo qual frequentemente era usada para fazer tábuas para cobrir casas e estábulos. Por ser pesada e dura, essa madeira foi muito procurada para dormentes de estradas de ferro e vigamentos de pontes. Por ser fácil de trabalhar, de aspecto agradável e de uma extraordinária durabilidade, é considerada como uma das madeiras mais valiosas do sul do Brasil.

Com base nestas informações, o presente trabalho foi realizado com a finalidade de ajustar e selecionar modelos matemáticos capazes de estimar o incremento periódico em área basal, o volume e biomassa de indivíduos jovens de Cabralea canjerana. Tais resultados podem vir a contribuir para futuras práticas no manejo sustentável desta espécie.

\section{MATERIAL E MÉTODOS}

O estudo foi desenvolvido em uma propriedade particular no município de Agudo, na região fisiográfica da 
Depressão Central do Rio Grande do Sul, coordenadas $29^{\circ} 30^{\prime} 0^{\prime \prime} \mathrm{S}$ e $53^{\circ} 10^{\prime} 14^{\prime \prime} \mathrm{O}$, com altitude média de $260 \mathrm{~m}$. O clima da região é do tipo Cfa, de acordo com a classificação de Köppen, com verões quentes e sem estação seca definida, a vegetação pertence ao domínio da Floresta Estacional Decidual e o solo é um Neossolo Litólico Eutrófico fragmentário (EMBRAPA, 2006).

A área sofreu um corte raso no início da década de 1990, com redução da biomassa residual pelo fogo. Logo após foi cultivada uma safra de milho, sem revolvimento do solo, seguido de pousio. A floresta no local contém árvores de grande porte no dossel e árvores pequenas no sub-bosque, com maioria dos indivíduos provenientes de brotação. Os indivíduos de Cabralea canjerana utilizados neste estudo encontravam-se no estrato intermediário da floresta e estavam, em geral, sob alta competição.

Para análise de crescimento foram utilizadas 19 árvores de Cabralea canjerana, das quais foram retirados discos transversais do fuste a 1,30 $\mathrm{m}$ do nível do solo DAP. Os discos foram secos em estufa a $40 \circ \mathrm{C}$ e posteriormente lixados. Os anéis de crescimento foram analisados e marcados no sentido medula-casca com auxílio de lupa. Logo após, os discos foram digitalizados e mensurados com o auxílio do Software Image Pro Plus4. Mediu-se o incremento em diâmetro dos dois últimos anos de crescimento, referentes a 2010 e 2011.

Para quantificar o volume (v), as árvores foram cortadas e seccionadas com o auxílio de um motosserra. Foram selecionadas e cubadas 12 árvores, com DAP mínimo de $5,2 \mathrm{~cm}$ e máximo de $11,0 \mathrm{~cm}$. No cálculo do volume rigoroso, empregou-se o método de Smalian.

Os dados de biomassa foram coletados utilizando-se o método destrutivo, fazendo-se uso de 14 árvores. Estas tiveram os componentes da biomassa acima do solo (Btotal), compartimentados em madeira (Bmad) e folhas (Bfol), mensurados a campo com o auxílio de uma balança de gancho digital, com precisão de 0,1 Kg. Para a amostragem da madeira foram retirados discos ao longo do tronco, sendo pesados no local e posteriormente transportados e secos em estufa a 40 으, até atingirem peso constante. As folhas amostradas passaram pelo mesmo processo de secagem e pesagem. A partir da relação peso seco e peso verde determinou-se o teor de umidade e foi realizado o cálculo da biomassa para cada componente, assim como do total.

Para a modelagem da estimativa das variáveis incremento periódico em área basal (IPg), volume (v), biomassa total (Btotal), biomassa da madeira (Bmad) e biomassa foliar (Bfol) foram testados seis modelos matemáticos (Tabela 1 ).
Tabela 1. Modelos matemáticos testados para diferentes variáveis de Cabralea canjerana.

\begin{tabular}{cc}
\hline Modelo Matemático & Autor \\
\hline$\hat{y}=b 0+b 1 . x$ & Linear \\
$\hat{y}=b 0+b 1 . x^{2}$ & Kopezky-Gehrhardt \\
$\hat{y}=b 0+b 1 . x^{2} z$ & Spurr \\
$\ln \hat{y}=b 0+b 1 . \ln x$ & Husch \\
$\hat{y}=b 0+b 1 . x+b 2 . x^{2}$ & Hohenadl-Krenn \\
$\ln \hat{y}=b 0+b 1 . \ln x+b 2 . \operatorname{lnz}$ & Schumacher-Hall \\
\hline
\end{tabular}

Para avaliação das equações ajustadas foi utilizada a menor soma dos escores dos critérios estatísticos: coeficiente de determinação $\left(R^{2}\right)$, erro padrão da estimativa (Sxy) e coeficiente de variação em percentagem (CV\%), além da análise gráfica da dispersão dos resíduos.

\section{RESULTADOS E DISCUSSÃO}

\section{Incremento periódico em área basal}

A relação do IPg com o DAP das árvores mostrou que quanto maior o DAP, maior foi o $\mathrm{IPg}$, de modo que se percebe o início da curva de incremento, devido ao fato dos indivíduos de Cabralea canjerana serem jovens e possuírem DAP entre 1,5 e $11,2 \mathrm{~cm}$. 0 incremento médio observado foi de $4,89 \mathrm{~cm}^{2}$, com valor mínimo de $1,10 \mathrm{~cm}^{2}$ e máximo de $12,03 \mathrm{~cm}^{2}$.

Os modelos matemáticos ajustados para estimativa do IPg explicaram pelo menos $70 \%$ da variação dos dados amostrados (Tabela 2). Somente a equação de Spurr obteve um coeficiente de determinação ajustado inferior a este valor ( $\left.R^{2} a j=0,584\right)$.

A equação com melhores estatísticas e menor soma dos escores foi a de Schumacher-Hall. A mesma apresentou melhor distribuição gráfica dos resíduos, resultando na equação final: InIPg=-0,149+1,777.InDAP-0,815.InHt.

Mattos (2007), estudando o incremento em $C$. canjerana observou que a espécie tem incremento em área basal ascendente à medida que apresenta maiores diâmetros. A equação ajustada para estimar o incremento em área basal apresentou estatísticas satisfatórias, com valores de $R^{2}=0,688$ e $S x y \%=39,9$. A equação definida no presente estudo obteve maior coeficiente de determinação e menor erro padrão da estimativa. 
Tabela 2. Estatística dos modelos ajustados para estimativa do IPg de Cabralea canjerana.

\begin{tabular}{ccccc}
\hline Modelo & b0 & b1 & b2 \\
\hline Linear & $-1,183$ & 0,939 & - \\
\hline Kopezky-Gehrhardt & 0,983 & 0,076 & - \\
Spurr & 1,780 & 0,006 & - \\
\hline Husch & $-0,475$ & 1,058 & - \\
\hline Hohenadl-Krenn & 2,098 & $-0,443$ & 0,110 \\
\hline Schumacher-Hall & $-0,149$ & 1,777 & $-0,815$ \\
\hline Modelo & $\mathbf{R}^{\mathbf{2}}$ aj & Syx & $\mathbf{C V} \%$ & Escores \\
\hline Linear & 0,704 & 3,2 & 38,9 & 12 \\
\hline Kopezky & 0,758 & 2,6 & 35,2 & 16 \\
\hline Spurr & 0,584 & 4,6 & 46,2 & 6 \\
\hline Husch & 0,780 & 3,7 & 25,8 & 10 \\
\hline Hohenadl & 0,749 & 2,6 & 35,8 & 15 \\
\hline Schumacher-Hall & 0,848 & 1,9 & 21,5 & 4 \\
\hline
\end{tabular}

\section{Ajuste de equações de volume}

$\mathrm{O}$ volume médio obtido nas árvores de canjerana foi de $0,0285 \mathrm{~m}^{3}$, com valores mínimo e máximo de 0,0105 $\mathrm{m}^{3}$ e $0,0445 \mathrm{~m}^{3}$, respectivamente. Todos os modelos testados para a estimativa do volume apresentaram estatísticas satisfatórias (Tabela 3).

Tabela 3. Estatística dos modelos ajustados para estimativa do volume de Cabralea canjerana.

\begin{tabular}{ccccc}
\hline Modelo & b0 & b1 & b2 \\
\hline Linear & $-0,01797$ & 0,00553 & \\
Kopezky-Gehrhardt & 0,00327 & 0,00034 & \\
\hline Spurr & 0,00298 & 0,00004 & \\
Husch & $-7,45970$ & 1,80683 & \\
\hline Hohenadl-Krenn & $-0,02989$ & 0,00870 & $-0,00020$ \\
\hline \multicolumn{2}{c}{ Schumacher-Hall } & $-9,38189$ & 1,58246 & 1,06384 \\
\hline Modelo & $\mathbf{R}^{\mathbf{2}}$ aj & Syx & $\mathbf{C V \%}$ & Escores \\
\hline Linear & 0,720 & $3,6 \mathrm{E}-05$ & 22,9 & 12 \\
\hline Kopezky & 0,705 & $3,8 \mathrm{E}-05$ & 23,5 & 16 \\
\hline Spurr & 0,870 & $1,7 \mathrm{E}-05$ & 15,6 & 6 \\
\hline Husch & 0,766 & $3,7 \mathrm{E}-05$ & 6,7 & 10 \\
\hline Hohenadl & 0,692 & $3,5 \mathrm{E}-05$ & 24,0 & 15 \\
\hline Schumacher-Hall & 0,902 & $1,8 \mathrm{E}-05$ & 4,3 & 4 \\
\hline
\end{tabular}

A equação com melhor desempenho foi a de Schumacher-Hall (InV= -9.382+1.583. InDAP+1.064.InHt), com coeficiente de determinação de 0,9 e coeficiente de variação de 4,3\%. Tais estatísticas evidenciam a eficiência e adequabilidade do modelo, com baixo erro padrão da estimativa (Syx) e alto coeficiente de determinação ajustado ( $\left.R^{2} a j\right)$. A equação apresentou boa distribuição dos resíduos.

É recomendado sempre que possível, para equações de volume, que a altura seja inserida no modelo com a finalidade de aumentar a precisão da estimativa do mesmo. O que foi verificado na presente análise, onde as duas equações com melhores estatísticas foram as de dupla entrada (Schumacher-Hall e Spurr) em função do DAP e altura.

\section{Ajuste de equação para estimativa da biomassa aérea total}

Conforme a árvore cresce em dimensão, a biomassa da mesma aumenta. A biomassa aérea total média foi de $12,72 \mathrm{~kg}$. A biomassa aérea mínima foi 0,41 kg e a máxima $28,55 \mathrm{~kg}$. Os modelos matemáticos ajustados para tal variável apresentaram bons coeficientes de determinação, superiores a 0,7 (Tabela 4).

Tabela 4. Estatística dos modelos ajustados para estimativa da biomassa aérea total de Cabralea canjerana

\begin{tabular}{|c|c|c|c|c|}
\hline \multicolumn{2}{|l|}{ Modelo } & bo & b1 & b2 \\
\hline \multicolumn{2}{|l|}{ Linear } & $-9,281$ & 2,831 & \\
\hline \multicolumn{2}{|c|}{ Kopezky-Gehrhardt } & $-0,822$ & 0,204 & \\
\hline \multicolumn{2}{|l|}{ Spurr } & $-0,502$ & 0,0208 & \\
\hline \multicolumn{2}{|l|}{ Husch } & $-2,847$ & 2,530 & \\
\hline \multicolumn{2}{|c|}{ Hohenadl-Krenn } & $-3,580$ & 0,887 & 0,142 \\
\hline \multicolumn{2}{|c|}{ Schumacher-Hall } & $-3,769$ & 1,779 & 1,131 \\
\hline Modelo & $R^{2} a j$ & Syx & CV\% & Escores \\
\hline Linear & 0,719 & 16,51 & 34,51 & 16 \\
\hline Kopezky & 0,730 & 15,84 & 33,81 & 12 \\
\hline Spurr & 0,891 & 10,66 & 21,44 & 9 \\
\hline Husch & 0,918 & 6,37 & 15,14 & 5 \\
\hline Hohenadl & 0,707 & 15,68 & 35,12 & 17 \\
\hline Schumacher-Hall & 0,954 & 6,93 & 11,29 & 4 \\
\hline
\end{tabular}

A equação com melhores estatísticas e menor soma dos escores foi a de Schumacher-Hall, com um alto coeficiente de determinação ajustado $\left(R^{2} a j=0,95\right)$ e menor coeficiente de variação (CV =11,29\%). A equação também apresentou a melhor distribuição gráfica dos resíduos.

Com a soma dos escores próxima ao modelo de Schumacher-Hall está o modelo de Husch com um escore de 5 pontos. A equação de Husch apresentou menor erro padrão da estimativa em relação à equação de 
Schumacher-Hall, porém o $\mathrm{R}^{2}$ aj. foi mais baixo e o CV\% foi maior. O modelo de Husch é um modelo matemático que possui somente uma variável independente (DAP), o que facilita sua aplicação em relação aos modelos de dupla entrada. Logo, é possível utilizar a equação de Husch ajustada para estimativa da biomassa de Canjerana com boa precisão quando não tem a variável altura das árvores.

No estudo da biomassa aérea de Ateleia glazeoviana (timbó), Fontoura et. al. (2017) testaram diferentes modelos alométricos para a estimativa da variável. 0 modelo de Schumacher-Hall apresentou alto $R^{2} a j$. $(0,919)$, contudo o erro padrão da estimativa em percentagem foi o maior dentre todas as equações testadas para a espécie. Sendo selecionada pelos autores a equação de Spurr para estimativa da biomassa aérea de timbó, que também utiliza como variáveis independentes o DAP e a altura.

\section{Ajuste de equação para estimativa da biomassa da madeira}

As árvores possuem em média $12,08 \mathrm{~kg}$ de biomassa no componente madeira. A menor biomassa foi de 0,36 $\mathrm{kg}$ e a maior de $27,40 \mathrm{~kg}$. O ajuste dos modelos para estimativa da biomassa encontra-se na Tabela 5.

Tabela 5. Estatísticas dos modelos ajustados para estimativa da biomassa da madeira de Cabralea canjerana

\begin{tabular}{ccccc}
\hline Modelo & b0 & b1 & b2 \\
\hline Linear & $-8,754$ & 2,680 & \\
\hline Kopezky-Gehrhardt & $-0,703$ & 0,193 & \\
Spurr & $-0,530$ & 0,020 & \\
Husch & $-3,009$ & 2,580 & \\
\hline Hohenadl-Krenn & $-4,045$ & 1,075 & 0,117 \\
\hline Schumacher-Hall & $-3,986$ & 1,784 & 1,198 \\
\hline Modelo & $\mathbf{R}^{\mathbf{2}}$ aj & Syx & $\mathbf{C V \%}$ & Escores \\
\hline Linear & 0,707 & 15,69 & 35,34 & 15 \\
\hline Kopezky & 0,714 & 15,29 & 34,97 & 12 \\
\hline Spurr & 0,891 & 9,84 & 21,55 & 7 \\
\hline Husch & 0,914 & 17,06 & 16,31 & 10 \\
\hline Hohenadl & 0,693 & 15,05 & 36,23 & 15 \\
\hline Schumacher-Hall & 0,953 & 10,44 & 12,00 & 4 \\
\hline
\end{tabular}

Novamente o modelo de Schumacher-Hall foi o que apresentou as melhores estatísticas e menor soma dos escores. $O$ mesmo possui alto valor de $\mathrm{R}^{2}$ aj., chegando a explicar $95 \%$ da variação dos dados de biomassa da madeira.

O modelo de Schumacher-Hall além de selecionado para estimativa da biomassa da madeira, foi selecionado para estimativas do incremento periódico em área basal (IPg), do volume (v) e da biomassa aérea das árvores de canjerana. Demonstrando que o mesmo é o que melhor representa essas variáveis, e ainda, que o DAP e a altura são informações necessárias para tais estimativas.

A altura da árvore pode ser uma variável de difícil mensuração a campo e, devido a isso, normalmente também é estimada com equações de regressão. No entanto, para árvores jovens como as do presente estudo, que se localizam no estrato intermediário da floresta, tal mensuração não encontra maiores dificuldades. Logo, sua aplicação como variável independente nas equações definidas é praticável e relativamente simples.

\section{Ajuste de equação para estimativa da biomassa foliar}

A biomassa foliar média foi de $0,64 \mathrm{~kg}$, com valores mínimos e máximo de 0,05 kg e 2,04 kg, respectivamente. Os modelos ajustados para esta variável não apresentaram boas estatísticas. Todos tiveram altos coeficientes de variação em percentagem e baixos coeficientes de determinação ajustados (Tabela 6).

Tabela 6. Estatística dos modelos ajustados para estimativa da biomassa foliar de Cabralea canjerana

\begin{tabular}{ccccc}
\hline Modelo & b0 & b1 & b2 \\
\hline Linear & $-0,528$ & 0,150 & \\
\hline Kopezky-Gehrhardt & $-0,121$ & 0,011 & \\
Spurr & 0,025 & 0,001 & \\
Husch & $-4,364$ & 1,773 & \\
\hline Hohenadl-Krenn & 0,468 & $-0,190$ & 0,025 \\
\hline Schumacher-Hall & $-4,386$ & 1,755 & 0,027 \\
\hline Modelo & $\mathbf{R}^{2}$ aj & Syx & CV\% & Escores \\
\hline Linear & 0,377 & 0,18 & 71,6 & 11 \\
\hline Kopezky & 0,438 & 0,16 & 68 & 6 \\
\hline Spurr & 0,345 & 0,19 & 73,4 & 14 \\
\hline Husch & 0,572 & 0,20 & 75,99 & 12 \\
\hline Hohenadl & 0,416 & 0,15 & 69,32 & 7 \\
\hline Schumacher-Hall & 0,533 & 0,19 & 79,4 & 12 \\
\hline
\end{tabular}

A equação com menor soma dos escores foi a de Kopezky, com um $\mathrm{R}^{2}$ aj. Igual a 0,438 , valor considerado baixo. A equação também apresentou elevado CV\%, no entanto é o menor valor dentre os modelos testados para 
esta variável. A equação final se apresenta $B f=0,121+0,011$. DAP ${ }^{2}$.

A biomassa foliar da canjerana variou muito entre árvores, pois algumas destas estavam em estágio de ráquis enquanto outras já haviam desenvolvido sua copa. Isto, aliado ao fato da espécie encontrar-se em uma floresta natural, onde está exposta a um ambiente heterogêneo e de alta competição, pode-se justificar a alta variabilidade da biomassa das folhas de canjerana e o baixo ajuste das equações de regressão.

Barbeiro et al. (2009) ao testar modelos matemáticos para gerar estimativas da biomassa de Nectandra grandiflora conseguiram ajustes muito bons para biomassa do fuste e biomassa total. No entanto, assim como no presente trabalho, os modelos testados para biomassa foliar tiveram baixo nível de precisão. Os autores atribuem tal resultado à irregularidade na arquitetura das copas dessa espécie, onde há grande heterogeneidade na biomassa foliar entre árvores de porte semelhante.

Cabe ressaltar que os resultados expostos no trabalho foram obtidos para árvores de pequenas dimensões (até $11,5 \mathrm{~cm}$ de DAP) e, portanto, os modelos ajustados são válidos somente para predições de árvores até a dimensão mencionada e que se apresentem sob condições similares de sítio e clima.

\section{CONCLUSÕES}

O modelo matemático de Schumacher-Hall obteve melhor ajuste nas estimativas de incremento periódico em área basal, volume, biomassas aérea total e da madeira.

As equações definidas para o incremento periódico em área basal, volume, biomassa aérea total e biomassa da madeira são, respectivamente:

$$
\begin{aligned}
& \text { InIPg=-0,149+1,777.InDAP-0,815. In Ht } \\
& \ln \mathrm{V}=-9.382+1.583 . \ln \mathrm{DAP}+1.064 . \ln \mathrm{Ht} \\
& \ln \mathrm{Bt}=-3,769+1,779 . \operatorname{lnDAP}+1,131 \mathrm{lnHt} \\
& \operatorname{lnBm}=-3,986+1,784 . \operatorname{lnDAP}+1,198 \mathrm{InHt}
\end{aligned}
$$

As equações para estimativa de biomassa foliar possuem estatísticas insatisfatórias com elevados valores de coeficiente de variação em percentagem.

\section{REFERÊNCIAS}

BARBEIRO, L.S.S. et al. Equações para estimativa da biomassa individual de Nectandra grandiflora Ness (Canela-amarela). Floresta, v.39, n.4, p.833-843, 2009.
CARVALHO, P.E.R. Espécies florestais brasileiras recomendações silviculturais, potencialidades e uso da madeira. Brasília: EMBRAPA/CNPF, 1994.

CHASSOT, T. Modelos de crescimento em diâmetro de árvores individuais de Araucaria angustifolia (Bertol.) Kuntze na Floresta Ombrófila Mista. 2009. 48p. (Dissertação de mestrado).

EMBRAPA. Empresa Brasileira de Pesquisa Agropecuária. Sistema de Classificação Brasileiro de Classificação de Solos. 2.ed. Rio de Janeiro: 2006.

FONTOURA, M.R. et al. Modelos alométricos para estimativa de biomassa em área de regeneração natural de Ateleia glazioviana baill. Floresta, v.47, n.4, p.469-478. 2017.

HOUGHTON, R.A. et al. Carbon dioxide Exchange between atmosphere and terrestrial ecosystems. In: Atmospheric carbono dioxide and the global carbon cycle. U. S., p.114-140. 1985.

LORENZI, H. Árvores brasileiras: manual de identificação e cultivo de plantas arbóreas nativas do Brasil. Nova Odessa: Plantarum, v.1, 1998.

MATTOS, R.B. Produtividade e incremento de Cabralea canjerana (Vell.) Mart., Cedrela fissilis Vell. e Cordia trichotoma (Vell.) Arrab. Ex Steud., em floresta nativa no Rio Grande do Sul. 2007. 106p. (Tese de doutorado).

PÁSCOA, F. et al. Estabelecimento simultâneo de equações de biomassa para o pinheiro bravo. In: Simpósio Iberoamericano de Gestión y Economía Forestal, Anais..., p.i-f, 2004.

REITZ, R. et al. Projeto madeira do Rio Grande do Sul. Porto Alegre: CORAG, 1983.

REITZ, R. et al. Projeto madeira do Rio Grande do Sul. Porto Alegre: Secretaria de Agricultura e Abastecimento, 1988.

SANTOS, E. Nossas Madeiras. Belo Horizonte. Ed. Itatiaia Ltda. 1987.

SCHNEIDER, P.R. Análise de regressão aplicada à engenharia florestal. Santa Maria: UFSM, 2008.

SILVA, J.N.M. Manejo florestal. Empresa Brasileira de Pesquisa Agropecuária. 2.ed. Brasília: Embrapa, 1996.

SILVEIRA, P. et al. Estado da arte na estimativa de biomassa e carbono em formações florestais. Floresta, v.38, n.1, p.185-206, 2008.

VACCARO, S. et al. Incremento em área basal de árvores de uma floresta estacional decidual, em três fases sucessionais, no município de Santa Tereza, RS. Ciência Florestal, v.13, n.2, p.131-142, 2003. 
BIOFIX Scientific Journal v. 3 n. 1 p. 96-102 2018

Lanzarin, K. et al.

WATZLAWICK, L.F. Estimativa de biomassa e carbono em

Floresta Ombrófila Mista e plantações florestais a partir de dados de imagens do satélite Ikonos II. 2003. 120p. (Tese de doutorado). 